\title{
Das Röteln-Syndrom
}

Vorbemerkungen zu den Referaten ernes Symposions

1941 beschrieb Gregg erstmals den teratogenen Effekt einer mütterlichen Rötelnerkrankung im ersten Schwangerschaftstri-menon. Seither haben die Fortschritte der Virusforschung wichtige Erkenntnisse zu diesem Problem geliefert. Besonders eindrucksvolle Untersuchungen waren möglich, als die Vereinigten Staaten von Amerika im Frühjahr und Sommer des Jahres 1964 die größte Röteln-Epidemie der vergangenen 20 Jahie erlebten. Die Zusam-menarbeit verschiedener klinischer und theoretischer Institute erbrachte eine große Zahl neuer Erkenntnisse zur Symptomatologie und Immunologie der Erkrankung. Auf einem gemeinsam von der Amerikanischen Pädiatrischen Gesellschaft und der Gesellschaft für pädiatrische Forschung am 6.5.1965 in Philadelphia veranstalteten Röteln-Symposion wurden die Ergebnisse der einzelnen Forscher-gruppen diskutiert. Dieser Tagung ist ein Sonderheft der «Ameri-kanischen Zeitschrift für Kinderkrankheiten» (Amer. J. Dis. Child) gewidmet, das durch einige Berichte über experimentelle Studien und prophylaktische Maßnahmen erweitert wurde.

Den Referaten eines Teiles dieser Arbeiten sollen zunächst einige in den Berichten häufig wiederkehrende klinische und im-munologische Daten vorangestellt werden:

Das Rubeolen-Syndrom beim Neugeborenen äußert sich häufi-ger als bislang bekannt und vermutet in einer thrombozytopeni-schen Purpura. Die weitere Symptomatik umfaßt eine Hepato-splenomegalie, kongenitale Herzvitien, angeborene Augenschäden (Katarakt, Glaukom, «wolkige» Cornea), Adenopathien, Knochen-schäden, Hepatitis und Anämien. Defekte des Urogenitalsystems werden dagegen nur selten beobachtet.

Die Häufigkeit von Spontanaborten nach mütterlicher Rötelnerkrankung ist doppelt so hoch wie die Durchschnittshäufigkeit. Das Geburtsgewicht erkrankter Kinder ist niedriger als das gesunder Neugeborener nach gleicher Schwangerschaftsdauer.

14 Gynäk. Rdsch., Bd. 3, Nr. 3 (1966)

194

Das Röteln-Syndrom

Virologische Untersuchungen wurden sowohl an verschiedenen Gewebeextrakten verstorbener Kinder als auch an Nasen-Rachen-abstrichen, Urin- und Stuhlproben und mit Blutseren lebender Personen vorgenommen. In geeigneten Fallen erfolgte der Nach-weis der Viren ferner im Fruchtwasser und an den Eihäuten. Die Methode zur Erfassung der Viren benutzt die Interferenz mit ECHO- oder Coxackie-Viren. Hierbei wird nach achttägiger In-kubation des Untersuchungsmaterials auf Affennierenkulturen der zytopathogene Effekt der zum Interferenznachweis herangezogenen Virusart geprüft.

Im Verlaufe einer Rötelninfektion sind grundsätzlich 2 Anti-körperarten nachweisbar:

neutralisierende Antikörper, deren Nachweis ebenfalls mit der Interferenztechnik vorgenommen wird und entsprechende Titer-angaben erlaubt, komplementfixierende Antikörper, deren Titer mit Hilfe eines hämolytischen Systems festgestellt werden kann. 
Die nachfolgenden Kondensate sind nicht in der Reihenfolge ihrer Veröffentlichung in dem angeführten Sonderheft wiedergegeben. Zur besseren Ubersicht erschien es zweckmäßiger, ihre Umgruppierung entsprechend der praktischen und theoretischen Bedeutung vorzunehmen. G. Oehlert

Aus: Amer. J. Dis. Child 110: 395-407 (1965); cit. Gynäk. Rdsch. 3: 194-196 (1966) Die Rötelnepidemie 1964

J. L. Sever, K. B. Nelson und M. R. Gilkeson, Bethesda, Md.

Die Veröffentlichung entstammt der Zusammenarbeit von 11 Krankenhäusern, die während und nach der Röteln-Epidemie 1964 über 6000 schwangere Frauen und ihre Neugebörenen beobach* Originaltitel: Rubella epidemic, 1964: Effect on 6000 pregnancies. I. Preliminary clinical and laboratory findings through the neonatal period; A report from the collaborative study on cerebral palsy. 